\title{
First-strand Library Sequencing
}

National Cancer Institute

\section{Source}

National Cancer Institute. First-strand Library Sequencing. NCI Thesaurus. Code C150428.

Directional sequencing, where the first read (or the only read in case of single end reads) is from the complement strand. 